\title{
Central neural influences on the human retina during selective attention
}

\author{
ROBERT G. EASON, MARTA OAKLEY, and LYNN FLOWERS \\ University of North Carolina, Greensboro, North Carolina
}

\begin{abstract}
Two controversial issues were investigated. One concerned the question of whether centrifugal fibers project to the human retina, and the other, the question of whether precortical filters which differentially transmit relevant and irrelevant information during selective attention exist. The issues were addressed jointly by studying the effects of selective attention on evoked responses obtained from the retina and over the occipital cortex. Selective attention was manipulated by requiring subjects to respond to stimuli presented at various locations in the visual field while attempting to ignore stimuli presented at other locations. Retinal responses to stimuli presented at attended to locations were found to be larger than those at unattended to locations. A similar result was obtained for responses recorded over the occipital cortex. The results are interpreted as follows: (1) They support the hypothesis that sustained voluntary attention to specific locations in space is mediated, at least partly, by centrally controlled centrifugal pathways which differentially modify synaptic transmission at a precortical level; (2) such differential alteration can occur as far peripherally as the retina; and (3) such alteration at the retinal level necessarily requires the existence of centrifugal optic nerve fibers. The results are discussed within the context of existing evidence bearing on the issues.
\end{abstract}

With the possible exception of the visual system, it is now generally accepted that central mechanisms exert centrifugal influences at an early stage of sensory transmission in the major sensory systems (Livingston, 1978). Since Cajal's early observations of efferent terminals in the retina (Polyak, 1957), numerous anatomical and physiological studies have provided supporting evidence for the existence of efferent fibers in the optic nerve and retina of vertebrates (Ogden, 1968; Van Hasselt, 1973), including humans (Honrubia \& Elliott, 1968; Sacks \& Lindenberg, 1969; Wolter, 1965; Wolter \& Lund, 1968). Estimates of the relative number of efferents range from $1 \%$ in birds (Cowan \& Powell, 1963) to up to $10 \%$ in humans (Wolter \& Lund, 1968) and other primates (Livingston, 1978). Except for the findings in birds (Cowan, 1970; Miles, 1972a, 1972b), the definitiveness of the evidence for the existence of such fibers in vertebrates, including humans, has been questioned (Brindley, 1970; Freund, 1973; Rodieck, 1973; Shortess, 1978; Singer, 1977).

Related to the issue of whether centrifugal fibers project via the optic nerve to the retina is the question of where in the visual system certain kinds of irrelevant or unattended information are filtered.

The results reported in this study were presented at the annual meetings of the Society for Neuroscience held in Los Angeles in 1981, and the Society for Psychophysiological Research held in Washington, D.C., 1981. The work was supported in part by UNC-G Grants $\mathbf{8 0 0 5}$ and $\mathbf{8 0 3 0}$. The author's mailing address is: Department of Psychology, University of North Carolina, Greensboro, North Carolina 27412.
Evidence obtained from animals indicates that centrifugal activity may alter the flow of incoming information as far peripherally as the spinal cord for the somatic sensory system (Coulter, Maunz, \& Willis, 1974) and the olivocochlear bundle for the auditory system (Rasmussen, 1960, 1964; Oatman, 1976; Oatman \& Anderson, 1977, 1980). Recently, Lukas $(1980,1981)$ reported data which strongly suggest that irrelevant auditory information may be filtered at the level of the olivocochlear bundle in humans, although other attempts to demonstrate such filtering have proved unsuccessful (Picton \& Hillyard, 1974; Picton, Hillyard, Galambos, \& Schiff, 1971; Picton, Stapells, \& Campbell, 1981).

The idea that unattended information may be filtered precortically was proposed over 2 decades ago by Hernandez-Peon, Scherrer, and Jouvet (1956), Lindsley $(1959,1961)$, Livingston (1959), and others, following the discovery of the ascending reticular activating system (Moruzzi \& Magoun, 1949) and of the existence of corticofugal pathways which can alter afferent transmission in specific thalamic relay nuclei via the thalamic reticular nucleus (French, Hernandez-Peon, \& Livingston, 1955; Negishi, Lu, \& Verzeano, 1962; Scheibel \& Scheibel, 1966, 1967). Numerous studies conducted on animals lend credibility to the precortical filtering hypothesis (Chalupa, Macadar, \& Lindsley, 1975; Coleman \& Lindsley, 1975, 1977; Gilbert \& Kelly, 1975; Lamarre, Filion, \& Cordeau, 1971; Livingston, 1978; Oatman, 1982; Singer, 1977; Skinner \& Yingling, 1977; Yingling \& Skinner, 1976; and others). 
Even though there is a significant amount of data from animal studies which support the existence of precortical filters, very few evoked-potential studies conducted with humans have provided evidence for their existence. Except for the findings of Lukas 1980,1981 ), the evidence that has been provided tends to be indirect and inferential in nature. Based on the observation that a relatively early negative component (now commonly referred to as N1) of the visually evoked potential (VEP) to peripherally presented light flashes was larger when the flashes were attended than when they were not, Eason, Harter, and White (1969) postulated that the effect could have resulted from precortical filtering of irrelevant information. Harter and Salmon (1972) postulated that a precortical filter could account for the attention effect on N1 that they observed when relevant stimuli of different colors and shapes were presented at the same spatial location. The intramodality attention effect observed on the N1 component of the auditory evoked potential (AEP) by Hillyard, Hink, Schwent, and Picton (1973) could also be interpreted as providing support for precortical filtering, although they did not impose such an interpretation on their data. Later studies by Eason (1981) and Van Voorhis and Hillyard (1977), in replications and extensions of the Eason et al. study (1969), demonstrated an attention effect on a very early positive component (P1), which preceded N1, further strengthening the case for precortical filtering of certain kinds of irrelevant information. ${ }^{1}$ Nonetheless, the possibility remains that those cortical areas receiving input from pathways conveying relevant and irrelevant information are differentially activated or "primed" by selective attention, and that the greater amplitude of the early EP components results from such differential cortical activation rather than from the differential transmission of relevant and irrelevant information through afferent pathways (Näätänen, 1975, 1982; Posner, Nissen, \& Ogden, 1977).

The present study attempts to resolve the peripheral filtering issue by providing a more direct test of the centrifugal feedback hypothesis in the human visual system than was employed in the VEP studies mentioned above. The test entails the recording of retinal activity along with VEPs obtained over the occipital cortex under differing selective attention instructions. A demonstration that the electrical response of the retina could be modulated by selectiveattention manipulations (1) would provide not only evidence for early neural filtering of irrelevant information, but also further evidence for the existence of centrifugal optic nerve fibers, and (2) indicate one important aspect of their functional significance.

The experimental paradigm was similar to that used in earlier VEP studies by Eason and his associates (Eason, 1981; Eason et al., 1969). Spatial location was chosen as the stimulus dimension along which to vary attention, as in the earlier studies, in order to minimize overlap in the neural channels activated by the attended and unattended stimuli. Selective attention was manipulated by requiring subjects to respond to stimuli appearing peripherally in one visual field while attempting to ignore and not respond to stimuli appearing concomitantly at the homologous location in the opposite field. An additional manipulation required the subjects to attend and respond to foveally presented stimuli while attempting to ignore and not respond to stimuli presented peripherally at homologous locations in both visual fields. The latter type of manipulation was found to be especially effective in an earlier study as a means of suppressing attention to irrelevant peripheral stimuli (Eason, 1981).

In addition to the effects of the attention manipulations on the retinal and occipital responses to peripheral stimulation, the effects of the visual field in which the stimuli appeared, as well as the eye stimulated, were examined.

\section{METHOD}

\section{Design}

The study was conducted in two phases as two separate experiments. The right eye was stimulated in the first experiment, and the left eye, in the second. Except for eye stimulated, the second experiment was a replication of the first.

Thirty-two subjects participated in the study, 16 in each experiment. Each subject participated in six trials, two under each of three attention conditions, during a single 2-h session. The last three trials were counterbalanced replications of the first three. Each lasted approximately $8 \mathrm{~min}$. The intertrial interval (ITI) was 5 min except at the midpoint of each session, at which time the subject took a 10 -min break. Under two of the conditions, the subjects (1) responded to stimuli appearing in one visual field (VF) while attempting to ignore those appearing in the opposite field, and (2) vice versa. Under the third condition, they responded to a foveal stimulus while attempting to ignore stimuli appearing concomitantly, but never simultaneously, in both visual fields. Order of presentation of conditions was varied across subjects (ABCCBA, ACBBCA, etc.).

In Experiment 1, responses were obtained with electrodes placed at the internal canthus of the right eye (RIC) and over the right occipital lobe $\left(\mathrm{O}_{2}\right)$. Analogous locations were used for lefteye stimulation ( $\mathrm{LIC}$ and $\mathrm{O}_{1}$ ) in Experiment 2. All recordings were monopolar, the right earlobe serving as reference. ${ }^{2}$ Since the longitudinal axis of a retinal dipole activated by a visual stimulus is oriented perpendicularly to the retinal area stimulated (Armington, 1974; Brown, 1968; Dowling, 1970; Eason, Flowers, \& Oakley, 1983), the right-eye electrode was in a more favorable position for recording responses to stimuli appearing in the left visual field (LVF) than in the right (RVF); the opposite was true for the lefteye electrode.

\footnotetext{
Subjects

Most of the subjects were advanced undergraduate and graduate students in their 20s; a few were nonstudents in their 30 s or early 40s. Two men and 14 women participated in Experiment 1, and 3 men and 13 women, in Experiment 2. None had previously participated in an evoked potential experiment, and all were naive as to the purpose of the study. All had normal or corrected vision. Those wearing corrective lenses were permitted to do so during data collection.
} 


\section{Apparatus and Procedure}

The physical parameters of the visual stimulus proved to be very critical in the manipulation of selective attention, and had to be selected carefully on the basis of results obtained in a series of parametric pilot studies. If the stimuli were too salient, due to physical size, intensity, or some combination of the two, pilot subjects had great difficulty attending to one stimulus while ignoring another. On the other hand, the reduction of saliency to the point that subjects had little difficulty differentially attending to relevant and irrelevant stimuli resulted in retinal responses too small to measure. The parameters used in the study constituted a compromise between those best suited for selectively attending to the stimuli and those required for evoking well-defined and easily measurable retinal potentials. The evoking stimuli consisted of $61 / 2$-deg flashed checkered patterns, each check subtending a visual angle of $30 \mathrm{~min}$ of $1 \mathrm{deg}$. The stimuli were presented $30 \mathrm{deg}$ peripherally in each visual field with Grass PS-2 photostimulators, and were of $10-\mu \mathrm{sec}$ duration. The flashes were approximately $2 \mathrm{log}$ units above a background luminance level of approximately $3 \mathrm{~mL}$.

During a trial, single and double flashes were presented randomly in each visual field, with the temporal constraint that both types of flash presentations be separated by at least $500 \mathrm{msec}$. The time between flashes varied from 0.6 to $2.0 \mathrm{sec}$, the median being about $1.0 \mathrm{sec}$. The double flashes, which were separated by $250 \mathrm{msec}$, were randomly interspersed among the single flashes and occurred approximately $25 \%$ of the time. Thus, the subject could not predict in which visual field the next stimulus would occur or whether it would be a single or double flash. After a few minutes of practice, each subject was able to detect the doublets (i.e., double flashes) with nearly perfect accuracy.

While selectively attending the peripherally located flashes in a given field, the subjects fixated the center of a circular display that subtended approximately $61 / 2 \mathrm{deg}$ of visual angle and had a brightness of $2 \log$ units above a background level of $3 \mathrm{~mL}$. When a given peripheral visual field (right or left) was relevant, the subject was required to count the number of times doublets appeared in that field and to report the number counted at the end of the run. Immediate feedback was given as to the accuracy of the count.

During the foveal attention condition, the subjects counted foveally presented numerals (digits) of a particular kind (e.g., 3s) embedded in a set of 12 numerals while attempting to ignore peripheral flashes appearing concomitantly in each visual field. During this task, they fixated the center of the same $61 / 2$-deg circular field used during peripheral attention conditions; however, in this condition rectangular displays of numerals arranged in three rows and four columns were presented. Each numeral subtended approximately $1 \mathrm{deg}$ of visual angle. The numerals appearing in each display were selected and arranged randomly. The displays were presented with a Kodak slide projector set automatically to present a new display approximately each second. The subjects maintained a running count of the number of times a particular numeral appeared in a block of 20 such displays. During a break of approximately $10 \mathrm{sec}$ following each block, the subjects reported their count and were given immediate feedback as to their accuracy. A different set of numerals were counted in each block of 20 displays. This procedure was repeated until the end of the trial, that is, until the number of flashes required to obtain an averaged evoked response had been presented.

The retinal and occipital responses were amplified by a Grass Model 7 polygraph equipped with 7P3 EEG preamplifiers, with the bandpass filters set to pass frequencies ranging from 1 to $35 \mathrm{~Hz}$. Averaged responses were obtained with a TMC signal averager over a 500 -msec epoch following each flash. Averages based on 100 single flashes from each visual field were obtained for each trial; responses to double flashes were not included in the average.

Subjects sat in an electrically shielded, light-controlled room. White noise masked clicks associated with the discharge of the photoflash units and any other extraneous sounds. Polygraphic recordings obtained during the course of a trial at the canthal electrode sites permitted continuous monitoring of eye movements, blinks, and other nonneural events. A 20-deg saccade in the horizontal plane produced a deflection in excess of $20 \mathrm{~mm}$; a full eye blink produced a deflection in excess of $30 \mathrm{~mm}$. Thus, such artifacts were easily discernible. As noted in an earlier study (Eason, 1981), subjects were able to maintain proper fixation throughout the course of each trial within the limits of normal physiological nystagmus.

Group analog averaged responses, based on 3,200 stimulus flashes, were obtained by combining across the two replications of averaged responses per subject per experimental condition in each of the two experiments. The latency of the peaks and troughs of the deflections contained in the group responses were used as an anchor point for establishing latency windows within which to make amplitude measurements of the deflections of each subject's averaged responses. The latency windows were set at $\pm 5 \mathrm{msec}$ about the latency values obtained from the group averaged responses. The vertical distance between the peak and trough of a given deflection, averaged across the 10 -msec latency window, constituted the quantitative measurement of that component. A data set for each condition consisted of 16 entries, one for each subject averaged across to replications.

\section{RESULTS}

\section{General Description and EP-Component Identification}

Group averaged responses of Experiment 1 are shown in the upper two quadrants of Figure 1, and those of the second experiment, in the lower two. Responses to flashes appearing in the LVF of the right eye are shown in the upper-left quadrant, and RVF responses, in the upper-right. The opposite arrangement holds for left-eye stimulation, RVF responses appearing in the lower-left quadrant and LVF responses in the lower-right. Attention conditions are depicted in the three segments of each quadrant. The labels above each segment denote whether subjects were attending to the left (LVF) or right (RVF) visual field or counting foveally presented numerals (digits). The responses shown in the left segment of each quadrant were obtained when the evoking flashes appearing in that particular field were task relevant, that is, when the subject counted double flashes appearing in that field. Those in each of the middle segments were obtained from the same field when the flashes appearing in the opposite field were task relevant. Those in each right segment were obtained from the same field when the foveal task was relevant. Retinal responses are shown in the upper row of each quadrant, and occipital responses are shown in the lower row. The retinal responses in the quadrants on the left were obtained under relatively favorable retinal-dipole-alignment conditions; those in the right quadrants were obtained under relatively unfavorable conditions (Eason et al., 1983).

The vertical lines shown on the tracings at various latencies represent the degree to which the amplitude of the deflections at those latencies varied across subjects, the length of each line representing 1 SD unit above and below the group average. 
The deflections subjected to quantitative analysis are identified in the first segment of the upper-left quadrant of Figure 1. The first discernible retinal component was of negative $(\mathrm{N})$ polarity and had an onset latency of approximately $10-12 \mathrm{msec}$ and a peak latency of approximately $20 \mathrm{msec}$ (B10-N20, where $\mathrm{B}=$ baseline). The latency and polarity of this component identifies it as the a-wave of the ERG (Armington, 1974; Eason et al., 1983). Because of its small size, no attempt was made to quantify and subject this component to statistical analysis. Also, there was no point in doing so, since the a-wave reflects presynaptic (receptor) activity (Armington, 1974; Brown, 1968) and cannot be influenced by centrifugal activity. The next deflection was a positive(P)going wave, with onset and peak latencies of approximately 20 and $60 \mathrm{msec}$, respectively (N20-P60). This deflection possesses latency and polarity characteristics of the b-wave of the ERG (Armington, 1974) and is so labeled. The next deflection was a negatively oriented one which frequently appeared to be a composite of two separate waves with different peak latencies but similar, if not identical, onset latencies of approximately $60 \mathrm{msec}$. The shorter wave peaked at approximately $90 \mathrm{msec}$ (P60-N90); the longer one peaked at approximately $120 \mathrm{msec}$ (P60-N120). The longer duration deflection matches closely the afterpotential of the ERG (Armington, 1974; Eason et al., 1983). For some subjects, the shorter deflection (P60-N90) appeared to be the leading edge of a biphasic deflection which "rode" on the longer P60-N120 component. Although not very apparent in the group tracings, sinusoidal oscillations beginning at about $150 \mathrm{msec}$ were very discernible in the retinal responses for some subjects.

In the occipital tracings (see second row of the upper-left quadrant of the figure), the earliest discernible deflection was of positive polarity and had an onset latency of about $40 \mathrm{msec}$ and a peak latency of $90 \mathrm{msec}$ (B40-P90); the second deflection, of negative polarity, had an onset latency of $90 \mathrm{msec}$ and a peak latency of $170 \mathrm{msec}$ (P90-N170); and the third deflection, of positive orientation, had an onset latency of $170 \mathrm{msec}$ and a peak latency of $200 \mathrm{msec}$ (N170-P200). Later sinusoidal oscillations, evident in the group tracings, appeared in the averaged responses of each subject.

\section{Effects of Experimental Manipulations}

Retinal responses. A three-way analysis of variance performed by computer, using the Bio-Med statistical package, on the combined data matrix of both experiments for each of the retinal deflections (192 scores per deflection) revealed several significant main effects and interactions. These findings, summarized in Table 1, are clearly manifested in Figure 1.

As reflected in the figure, the retinal responses to flashes appearing $30 \mathrm{deg}$ peripherally in a given visual field (see, for example, LVF responses depicted in upper-left quadrant) were of largest magnitude when that field was task relevant, of intermediate size when the opposite field was relevant, and smallest when the foveal area was relevant. Retinal responses were larger when the recording electrode was relatively favorably aligned with the longitudinal axis of the retinal dipole (compare canthal recordings in the left two quadrants with those in the right two). Larger responses were obtained from the right eye than from the left (compare responses in upper quadrants with those in lower quadrants). The degree of change in the amplitude of the b-wave component (N20-P60) across the three attention conditions was greater for the favorable than for the unfavorable dipole orientation, as reflected by the significant attention $\times$ dipole interaction indicated in the table (compare changes across segments in the left quadrants with those in the right quadrants). The attention manipulation had a greater effect on the b-wave and P60-N120 components of the responses obtained from the right than on those from the left eye, as reflected by the significant dipole $x$ eye interactions (compare attention effects in upper quadrants with those in lower quadrants). The effect of dipole orientation on each of the retinal measures was greater for the right than for the left eye, as reflected in the significant dipole $x$ eye interactions (for each measure, compare differences in magnitude between the two upper quadrants with differences between the lower two).

Post hoc analyses revealed that significant dif-

Table 1

Statistical Summary of Retinal Effects

\begin{tabular}{|c|c|c|c|c|c|c|c|c|c|}
\hline \multirow{3}{*}{$\begin{array}{l}\text { Source of } \\
\text { Variation } \\
\end{array}$} & \multicolumn{9}{|c|}{ Deflections } \\
\hline & \multicolumn{3}{|c|}{ N20-P60 } & \multicolumn{3}{|c|}{$\mathrm{P} 60-\mathrm{N} 90$} & \multicolumn{3}{|c|}{ P60-N120 } \\
\hline & $\mathrm{F}$ & df & $\mathrm{p}<$ & $\mathrm{F}$ & $\mathrm{df}$ & $\mathrm{p}<$ & $\mathrm{F}$ & df & $\mathrm{p}<$ \\
\hline Attention & 5.44 & 2,60 & .01 & 16.81 & 2,60 & .001 & 17.32 & 2,60 & .001 \\
\hline Dipole Orientation & 69.44 & 1,30 & .001 & 19.45 & 1,30 & .001 & 65.08 & 1,30 & .001 \\
\hline Eye Stimulated & 14.01 & 1,30 & .001 & 4.77 & 1,30 & .05 & 17.42 & 1,30 & .001 \\
\hline Attention $\times$ Dipole & 4.80 & 2,60 & .01 & .48 & 2,60 & & .05 & 2,60 & \\
\hline Attention $\times$ Eye & 8.09 & 2,60 & .01 & 2.13 & 2,60 & & 4.46 & 2,60 & .02 \\
\hline Dipole $\times$ Eye & 24.00 & 1,30 & .001 & 12.54 & 1,30 & .01 & 12.45 & 1,30 & .001 \\
\hline
\end{tabular}



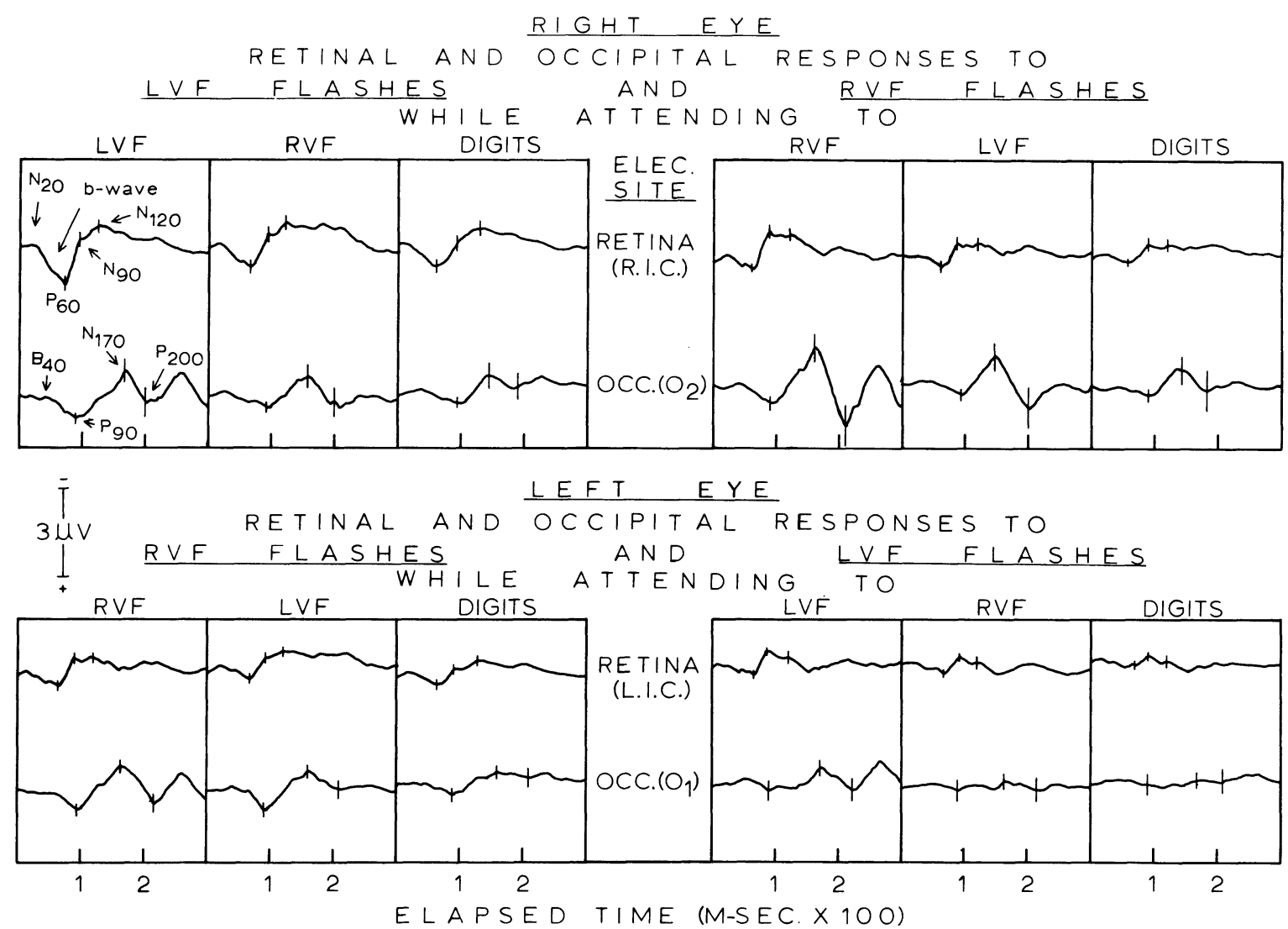

Figure 1. Retinal and occipital responses to flashes presented $30 \mathrm{deg}$ peripherally from within a given visual field (right or left) when subjects (a) attended to the location of the flashes in that field, (b) attended to the location of the flashes in the opposite field, or (c) attended to the location of foveally presented stimuli. These three conditions are depicted, respectively, in the left, middle, and right segments of each quadrant. Responses to righteye stimulation are shown in the top quadrants, and those to left-eye stimulation, in the lower quadrants. Each tracing is the grand average of 32 averaged responses obtained from 16 subjects (two replications per subject), and is based on a total of 3,200 stimulus flashes (100 per inividual average). Negativity is up in all tracings. See text for further details.

ferences between pairs of means of the b-wave component for the three attention conditions were limited to the right eye, but that for the other two components (P60-N90 and P60-N120) the attention effect was significant for each eye separately. A quadrant by quadrant variance analysis of each component (based on 48 scores per component constituting the data matrix of a given quadrant in Figure 1) indicated that the main attention effect noted in the comprehensive analysis for the b-wave was due primarily to results obtained from the right eye under the favored dipole orientation (upper-left quadrant of Figure 1). The attention manipulation for this quadrant was highly significant $[F(2,30)=9.35, p<.01]$, whereas the effect of the manipulation on the other quadrants, taken separately, was not. Nonetheless, the trend for all quadrants was the same, and the changes noted in each collectively contributed to the main effect obtained in the comprehensive analysis.

In summary, all three retinal deflections were sig- nificantly affected by the attention manipulation, the effect being more pronounced for the right eye than for the left, and for the favored than for the unfavored dipole orientation. Also, larger responses were obtained from the right than from the left eye.

Occipital responses. A comprehensive ANOVA performed on the entire data matrix of each of the VEP-components obtained over the occipital cortex (192 scores per component) revealed a significant attention main effect for P90-N170 $[\mathrm{F}(2,60)=9.56$, $\mathrm{p}<.001]$ and N170-P200 $[\mathrm{F}(2,60)=14.01, \mathrm{p}<$ $.001]$. The effect on the very early positive component (B40-P90) approached significance at the .05 level $[F(2,60)=2.76, p<.07]$. The attention effect did not interact significantly with eye stimulated, and none of the measures differed significantly as a function of eye stimulated. Significant lobe $\times$ eye interactions were obtained for $\mathrm{P} 90-\mathrm{N} 170[\mathrm{~F}(1,30)=$ $8.64, p<.01]$ and for N170-P200 $[F(1,30)=16.21$, $\mathrm{p}<.001]$. No other significant interactions were obtained. 
Separate variance analyses of the B40-P90 component performed on the data of each eye separately $(\mathrm{N}=96)$ revealed a significant attention effect for the right eye $[F(2,30)=4.24, p<.025]$ but not for the left. Thus, the nearly significant main effect for this component in the comprehensive analysis summarized above was due primarily to the right-eye data. Quadrant by quadrant analyses performed on the data matrices of the P90-N170 component revealed significant attention effects in all cases except for the left-eye/LVF condition (lower-right quadrant of Figure 1) [for the upper-left quadrant, $F(2,30)$ $=3.76, p<.05$; for the upper-right quadrant, $F(2,30)=4.61, p<.025$; and for the lower-left quadrant, $F(2,30)=7.74, p<.01]$. Similar analyses performed on the N170-P200 component revealed significant effects for every quadrant [for the upperleft, $F(2,30)=3.57, \mathrm{p}<.05$; for the upper-right, $F(2,30)=3.24, p<.001$; for the lower-left, $F(2,30)$ $=13.91, \mathrm{p}<.001$; for the lower-right, $F(2,30)=4.14$, $\mathrm{p}<.05]$.

\section{DISCUSSION}

\section{Retinal Effects}

The results of this study indicate that the amplitude of the responses obtained to peripherally presented flashes at canthal recording sites was influenced by the experimental manipulations designed to alter direction of sustained attention. In general, response amplitude was found to be greater when a given peripheral location was relevant to a one- vs. two-flash discrimination task than when it was not. Also, the attention effect was greater, the more favorably aligned the canthal electrodes were with the radial axis of the retinal area stimulated.

These results support the hypothesis that sustained voluntary attention to specific locations in space is at least partly mediated by centrally controlled centrifugal pathways which differentially modify synaptic transmission at a precortical level. Furthermore, the results indicate that this differential alteration can occur as far peripherally in the visual system as the retina. Such alteration, of necessity, would require the existence of centrifugal fibers projecting to the retina, and the results may be viewed as providing further evidence of their existence and as indicating one aspect of their functional significance.

The validity of these conclusions rests in part on whether the measured components of the responses obtained at the internal canthi, relative to the earlobe, were in fact of retinal origin. Two lines of evidence indicate that at least two of the components, $\mathrm{N} 20-\mathrm{P} 60$ and P60-N120, referred to earlier as the "bwave" and "afterpotential" of the ERG, were generated in the retina. The first is that the latency and polarity of the components are comparable to the bwave and afterpotential observed in corneally recorded ERGs of humans under similar luminance and background conditions (Armington, 1974). The second comes in part from the present study as well as from another, recent study conducted by the authors (Eason et al., 1983), wherein the amplitude of these two components was found to depend on the angular orientation of the recording electrodes relative to the radial axis of the retinal area stimulated. Since the longitudinal axis of the dipole source giving rise to the ERG is oriented perpendicularly, that is, radially, to the retinal surface (Brindley, 1970; Brown, 1968; Rodieck, 1973), its orientation relative to a canthal electrode can be easily altered by rotating the eyes to various positions while holding constant the retinal area stimulated. Eason et al. (1983) found that such variations in eye rotation altered dramatically both the amplitude and polarity of the N20-P60 and P60-N120 components contained in canthal recordings, indicating that they were of retinal origin. The findings of the present study, (1) that the deflections were of larger amplitude when the canthal electrodes were more favorably aligned with the radial axis of the retinal area stimulated than when they were not, and (2) that the attention effect was more pronounced under the more favorably aligned condition, provide further support.

There is the remote possibility that the earlobe reference electrode was not totally neutral with respect to the dipole source or sources responsible for the fluctuations in magnitude of the b-wave and afterpotential, and that early electrical activity occurring somewhere else in the visual system summated with the ERG components to produce the attention effects. This possibility seems extremely unlikely in view of the fact that no discernible effects were observed as a function of which earlobe was used as reference in a pilot study. Furthermore, pilot recordings between earlobes resulted in virtually flat lines.

To our knowledge, this study constitutes the first demonstration that manipulation of selective attention to different points in space affects sensory transmission at the retinal level in humans. However, Mirsky (1978) reported that the b-wave of the ERG to light flashes recorded from a patient was smaller during spike-wave EEG activity than during nonspike-wave activity. He suggested the reduced ERG may have resulted from a loss of concentration or attention to the flashes during the time the patient was experiencing a petit mal (spike-wave activity) seizure. Mirsky, Bloch, Tecce, Lessell, and Marcus (1973) also observed a reduction in the b-wave to an evoking stimulus during experimentally induced spike-wave activity in monkeys which they interpreted as possibly being due to loss of attention. Our results support their interpretation. In addition, the results are consistent with findings of Jacobson and 
Gestring (1958) that the ERG of cats and monkeys is depressed by administration of Metrazol, hexamethonium, or stimulation of the reticular formation, and that section of the optic nerve eliminates these effects. They concluded that a center exists in the brain which controls retinal function and that its influence is manifested in the ERG.

The effects noted in the present study also are consistent with those reported by Lukas for the human auditory system $(1980,1981)$. He observed that the auditory nerve component of the AEP was lower in amplitude and of longer latency to unattended than to attended tone pips. He interpreted these effects as probably being due to efferent influences of the olivocochlear bundle (OCB), since findings in animals had indicated that this structure has an inhibitory effect on auditory nerve activity (Desmedt, 1962, 1975; Dewson, 1967, 1968; Galambos, 1956; Klinke \& Galley, 1974; Starr \& Wernick, 1968; Wiederhold \& Kiang, 1970). Also, studies by Oatman and his associates (Glenn \& Oatman, 1977; Oatman, 1971, 1976; Oatman \& Anderson, 1977, 1980) have shown that evoked responses to tone bursts at the level of the cochlear nucleus are attenuated when unanesthetized cats attend to a visual stimulus, further implicating the $\mathrm{OCB}$ as playing an inhibitory role at the level of the cochlear nucleus and auditory nerve.

McCallum, Curry, Cooper, Pocock, and Papakostopoulos (1983) have just reported an observation consistent with the view that auditory information pertaining to spatial location may be filtered precortically, although these investigators did not impose such an interpretation on their data. They found that an early component with an onset latency of $26 \mathrm{msec}$, and a difference potential with an onset latency as short as $15 \mathrm{msec}$, were influenced by instructions to respond to sounds coming from a particular speaker among an array of four speakers located in front, behind, and on either side of the subject. The latencies of these components fell within the range of medium latency AEP deflections believed to arise, in part, from the thalamus (Picton, Hillyard, Krausz, \& Galambos, 1974), suggesting that the modulation of the components might be due to filtering at that level.

The above findings for the auditory system, in conjunction with the well-established fact that centrifugal fibers project to and modulate afferent activity at very early synaptic relays of the proprioceptive (Matthews, 1964), olfactory (Cragg, 1962; Gervais, 1979; Kerr \& Hagbarth, 1955; Shepherd, 1970), and somatic (Coulter et al., 1974; Livingston, 1978; Oleson, Ashe, \& Weinberger, 1975; Schmidt, 1973) sensory systems lend credibility to the results obtained in the present study for the visual system.

Even though definitive proof of the existence of centrifugal optic nerve fibers has been difficult to establish (Brindley, 1970; Livingston, 1978; Rodieck, 1973), and was questioned in recent reviews (Singer, 1977; Shortess, 1978), anatomical and functional evidence for the existence of such fibers in vertebrates ranging from fish (Ebbesson \& Meyer, 1981) to humans (Livingston, 1978) continues to accumulate. Using horseradish peroxidase (HRP), Halpern, Wang, and Colman (1976) demonstrated that such fibers project to the eye of the snake; also, there is strong physiological evidence for the existence of fibers in turtles (Marchiafava, 1976) and frogs (Byzov \& Utina, 1971). The existence of centrifugal fibers in birds has been well established (Miles, 1972a, 1972b). Among mammals, there is evidence that centrifugal fibers exist in the optic nerve of mice (Goldberg \& Galin, 1973), cats (Brook, Downer, \& Powell, 1965; Granit, 1955, 1959; Jacobson \& Gestring, 1958; Spinelli, Pribram, \& Weingarten, 1965), monkeys (Brook et al., 1965; Jacobson \& Gestring, 1958; Mirsky et al., 1973; Nobak \& Mettler, 1973), and humans (Honrubia \& Elliott, 1968; Mirsky, 1978; Sacks \& Lindenberg, 1969; Van Hasselt, 1972/73; Wolter, 1965; Wolter \& Lund, 1968). The results of the present study add another increment to the list.

There is little information as to the source of origin of retinopetal fibers. Anatomical studies in monkeys (Brook et al., 1965; Nobak \& Mettler, 1973) suggest that the fibers originate in the tectal region and pass through the midbrain to the optic tract. After passing through the chiasm, they traverse the central part of the optic nerve to the retina. Granit (1955) provided functional evidence that at least some of the fibers might originate in the reticular formation. Since it is known that corticofugal fibers project from visual cortical areas to the dorsal lateral geniculate nucleus (LGNd; Singer, 1977), the pulvinar (Wilson, 1978), the superior colliculus (Goldberg \& Robinson, 1978; Wurtz \& Albano, 1980), the reticular formation (Singer, 1977), and other mesencephalic nuclei (Rodieck, 1979), it seems plausible that the dendrites of the retinopetal fibers may lie in close proximity to one or more of these structures. The hypothalamus is another possibility, since it is known that the hippocampus, which has been shown to be involved in changes in attentional states in cats (Coleman \& Lindsley, 1975, 1977; Oatman, 1982), projects to that structure as well as to the reticular formation (Nauta, 1958).

\section{Occipital effects}

The attention manipulations significantly affected the early positive component (B40-P90, hereafter referred to as P1) of VEPs obtained over the occipital cortex when the right eye was stimulated. However, the effect of attention was not significant for the left eye, and only approached significance in an analysis of the data matrix for the two eyes combined $(p<$ 
.07). Since statistical significance was limited to the right eye, it is apparent that the attention effect on P1 was not as robust as in an earlier study (Eason, 1981) which had used less salient stimuli. The effect of the attention manipulations on the later components (P90-N170 and N170-P200) were significant for each eye separately and for the two eyes combined. Thus, the results are in general accord with, and tend to corroborate, the attention effects noted in earlier experiments (Eason, 1981; Harter, Aine, \& Schroeder, 1982; Van Voorhis \& Hillyard, 1977), with the exception that Van Voorhis and Hillyard did not observe a significant attention effect on P90-N170 (N1).

As stated earlier, the attention effects observed on early VEP components recorded over occipital cortex cannot be viewed as definitive evidence of precortical filtering. Nonetheless, a comparison of the latencies of these components with response latencies obtained from recordings of single neurons in striate cortex, the LGNd, and superior colliculus of animals may provide important temporal cues in relation to this issue. In the present study, which utilized relatively intense stimuli in order to obtain measurable ERGs, the onset latency of P1 was approximately $40 \mathrm{msec}$. In studies using less intense stimuli, relative to the background, onset latency was approximately $70 \mathrm{msec}$ (Eason, 1981; Van Voorhis \& Hillyard, 1977). Harter et al. (1982) obtained P1 onset latencies of approximately $80 \mathrm{msec}$ in recordings from central head regions lateral to the vertex.

The onset latency of P1 observed in the present and previous studies overlaps that obtained from cat striate cortex to diffuse light flashes under nearthreshold conditions (Creutzfeldt \& Kuhnt, 1973). Based on simultaneous recordings of VEPs from striate cortex and from single cells at various depths in the cortex, Creutzfeldt, Rosina, Ito, and Probst (1969) obtained data indicating that the early positive component of the VEP might be a manifestation of activity in geniculostriatal terminals along with postsynaptic activity (excitatory, then inhibitory) in striatal cells located in the deeper layers of the cortex. It seems plausible that the scalp-recorded P1 over the occipital cortex could arise in part from such activity. Similarly, more frontal recordings of P1 could arise from thalamocortical and cortical activity in the deeper layers of other cortical areas, including extrastriatal areas which receive projections from the pulvinar (Wilson, 1978).

Observations on the latency and duration of singleunit responses in the cat LGN suggest that the scalprecorded P1 may be, in part, a "far-field" recording of thalamic activity. Baker, Sanseverino, Lamarre, and Poggio (1969) found that stimulation of the cat's eye with small spots of light at near-threshold intensity resulted in an onset latency of approximately $55 \mathrm{msec}$; at higher intensities (log contrast-1.5), onset latency was about $25 \mathrm{msec}$. Peak latencies for near threshold and for higher intensity flashes varied from approximately 100 down to $60 \mathrm{msec}$. The response duration of the units was $150 \mathrm{msec}$ or more for a wide range of flash intensities, depending on whether a unit was of the "extended type" or "rebound type." Similar latency data were obtained by Negishi et al. (1962). Although the temporal overlap of LGN activity with scalp-recorded P1 does not constitute proof that the latter may be a "far-field" reflection of thalamic activity, it is most interesting that LGN neurons are maximally active at the time P1 occurs at occipital and central scalp locations.

Another type of "evidence" suggesting that P1 may reflect thalamic activity is provided by selective attention studies by Skinner and Yingling (1977) and Yingling and Skinner (1976) on cats. Based on a review of the literature and their own experiments, these investigators have proposed that the reticular nucleus of the thalamus operates as a "topographically organized inhibitory gate" that can modulate the flow of information through specific relay nuclei of the thalamus, thereby controlling the selective ascent of sensory input to the cortex.

\section{Glial Cell Contributions to Retinal Responses}

Since observations on the mudpuppy indicate that the b-wave of the ERG arises from the depolarization of Müller (glial) cells (Miller \& Dowling, 1970), its modulation by attention manipulations merits discussion. It is known that Müller cells depolarize in response to an increase in potassium ions $\left(\mathrm{K}^{+}\right)$in extracellular space, and that such an increase results from neuronal activity (Kuffler, Nicholls, \& Orkand, 1966). Also, it is known that synchronous optic nerve activity produces depolarization of Müller cells lying in close proximity to activated neurons (Miller \& Dowling, 1970). Recently, Miller, Dacheux, and Proenza (1977) demonstrated that antidromic stimulation of the optic nerve of axolotl (Mexican salamander) results in depolarization of Müller cells in the retina. They also found that depolarization produced by optic-nerve stimulation reduces the glial cell response to light stimulation, and that the presentation of a light stimulus just prior to the opticnerve stimulus decreases the magnitude of the antidromic response. From these observations, it seems clear that the b-wave of the ERG reflects the degree of activation of neurons, including ganglion cells, in the human retina, even though it does so indirectly via glial cell activity (Armington, 1974; Eason et al., 1983; Miller et al., 1977).

A comparison of the onset latency of the b-wave to the onset latency of evoked activity in more central structures strengthens the above interpretation of the neural significance of the b-wave. Recall that in the present study the onset latency of the b-wave, as measured at the apex of the a-wave, was approximately $20 \mathrm{msec}$, and that the onset latency of cat LGN neurons was $25-50 \mathrm{msec}$, depending on stimulus contrast (Baker et al., 1969). The latency of cells in 
the superior colliculus to spots of light have been found to vary from 40 to $80 \mathrm{msec}$, depending on stimulus parameters and retinal area stimulated (Wurtz \& Albano, 1980). The longer latencies obtained at the more central locations, in comparison with the onset of the b-wave, are consistent with the view that the $b$-wave reflects evoked neural activity in the retina which precedes the onset of tectal and thalamic evoked activity.

\section{Replicability}

Although not an exact replication, since different eyes and subjects were used, the second experiment of this study constitutes a quasi-replication of the first in that the same paradigm was employed. Two of the retinal components (P60-N90 and P60-N120) were significantly affected by the attention manipulations in the second experiment, confirming the effect obtained for these components in the first experiment. Although, in the second experiment, the bwave changed in the expected direction as a function of the attention conditions, it did not reach statistical significance. Even so, the fact that the two later components were significantly affected in both experiments, including P60-N120 (the afterpotential), which is known to be of retinal origin (Armington, 1974; Eason et al., 1983), provides confirming evidence for the existence of centrifugal optic-nerve fibers.

Since replication by others of the attention effects demonstrated in this study will crucially depend on the selection of stimulus parameters, it seems important to reiterate a point made in the methods section. That is, when ERGs are recorded with canthal electrodes, as in the present study, the use of near-threshold stimulus parameters, which maximizes selective attention, results in immeasurable ERGs. Thus, a compromixe is required wherein stimulus saliency must be sufficiently high to elicit measurable ERGs, yet sufficiently low to permit selective attention. This methodological problem may be avoided with the use of corneal electrodes, since corneal recordings are magnitudes greater than those obtained at canthal locations (Armington, 1974; Eason et al., 1983).

\section{Summary and Conclusion}

The principal finding of this study was that when one selectively attends to different locations in the visual field, retinal responses recorded at the internal canthi of each eye are larger when a given location is attended to than when it is not. This finding was obtained for both the b-wave and afterpotential of the ERG, as well as for a component which "rode" on the afterpotential. A second finding of significance was that an early positive component of the VEP recorded over the occipital cortex was affected by the attention manipulations, corroborating results obtained in earlier experiments (Eason, 1981; Harter et al., 1982; Van Voorhis, 1977). The effect observed on the retinal recordings is interpreted as functional evidence for the existence of centrifugal optic-nerve fibers that terminate in the retina, as well as evidence for the existence of a sustained, prestimulus, selective attention mechanism that results in the early peripheral filtering of irrelevant information pertaining to spatial location.

\section{REFERENCES}

Armington, J. C. The electroretinogram. New York: Academic Press, 1974.

Baker, F. H., Sanseverino, E. R., Lamarke, Y., \& Poggio, G. F. Excitatory responses of geniculate neuroris of the cat. Journal of Neurophysiology, 1969, 32, 916-929.

Brindey, G. S. Physiology of the retina and visual pathway (2nd ed.). London: Arnold, 1970.

Brooke, R. N. L., Downer, J., \& Powell, T. P. S. Centrifugal fibres to the retina in the monkey and cat. Nature, 1965, 207, 1365-1367.

Brown, K. T. The electroretinogram: Its components and their origins. Vision Research, 1968, 8, 633-677.

Byzov, A. L., \& Utina, I. A. Centrifugal effects of amacrine cells in the frog's retina. Neurophysiology, 1971, 3, 219-224.

Chalupa, L. M., Macadar, A. W., \& Lindsley, D. B. Response plasticity of lateral geniculate neurons during and after pairing of auditory and visual stimuli. Science, 1975, 190, 290-292.

Coleman, J. R., \& Lindsley, D. B. Hippocampal electrical correlates of free behavior and behavior induced by stimulation of two hypothalamic-hippocampal systems in the cat. Experimental Neurology, 1975, 49, 506-528.

Coleman, J. R., \& LindsLeY, D. B. Behavioral and hippocampal electrical changes during operant learning in cats and effects of stimulating two hypothalamic-hippocampal systems. Electroencephalography \& Clinical Neurophysiology, 1977, 42, 309-331.

Coulter, J. D., Maunz, R. A., \& Willis, W. D. Effects of stimulation of sensorimotor cortex on primate spinothalamic neurons. Brain Research, 1974, 65, 351-356.

Cowan, W. M. Centrifugal fibres to the avian retina. British Medical Bulletin, 1970, 26, 112-118.

Cowan, W. M., \& Powell, T. P. S. Centrifugal fibers in the avian system. Proceedings of the Royal Society of Britain, 1963, 158, 232-252.

CragG, B. G. Centrifugal fibers to the retina and olfactory bulb, and composition of the supraoptic commissures in the rabbit. Experimental Neurology, 1962, 5, 406-427.

CreutzFeldT, O. D., \& KuhNT, U. Electrophysiology and topographical distribution of visual evoked potentials in animals. In R. Jung (Ed.), Handbook of sensory physiology (Vol. VII/3): Central processing of visual information. Berlin: Springer-Verlag, 1973.

Creutzfeldt, O. D., Rosina, A., Ito, M., \& Probst, W. Visual evoked response of single cells and of the EEG in primary visual area of cat. Journal of Physiology, 1969, 32, 127-139.

Desmedt, J. E. Auditory-evoked potentials from cochlea to cortex as influenced by activation of the efferent olivocochlear bundle. Journal of the Acoustical Society of America, 1962, 34, 1478-1496.

DeSmedT, J. E. Physiological studies of the efferent recurrent auditory system. In W. D. Keidel \& W. D. Neff (Eds.), Handbook of sensory physiology (Vol. 5). Berlin: Springer-Verlag, 1975.

Dewson, J. H., III. Efferent olivocochlear bundle, some relationships to noise masking and to stimulus attenuation. Journal of Neurophysiology, 1967, 30, 817-832.

Dewson, J. H., III. Efferent olivocochlear bundle, some re- 
lationships to stimulus discrimination in noise. Journal of Neurophysiology, 1968, 31, 122-130.

Dowling, J. E. Organization of vertebrate retinas. Investigative Ophthalmology, 1970, 9, 655-680.

EAson, R. G. Visual evoked potential correlates of early neural filtering during selective attention. Bulletin of the Psychonomic Society, 1981, 18, 203-206.

Eason, R. G., Flowers, L., \& OAkley, M. Differentiation of retinal and nonretinal contributions to averaged evoked responses obtained with electrodes placed near the eyes. Behavior Research Methods \& Instrumentation, 1983, 15, 13-21.

Eason, R. G., Harter, M. R., \& White, C. T. Effects of attention and arousal on visually evoked cortical potentials and reaction time in man. Physiology \& Behavior, 1969, 4, 283-289.

Ebbesson, S. O. E., \& Meyer, D. L. Efferents to the retina have multiple sources in teleost fish. Science, 1981, 214, 924-926.

French, J. D., Hernandez-Peon, R., \& Livingston, R. B. Projections from cortex to cephalic brain stem (reticular formation) in monkey. Journal of Neurophysiology, 1955, 18, 44-55 \& 74-95.

FreUnd, H.-J. Neuronal mechanisms of the lateral geniculate body. In R. Jung (Ed.), Handbook of sensory physiology (Vol. VII/3): Central processing of visual information. Berlin: Springer-Verlag, 1973.

Galambos, R. Suppression of auditory nerve activity by stimulation of efferent fibers to cochlea. Journal of Neurophysiology, $1956,19,424-437$.

Gervais, R. Unilateral lesions of the olfactory tubercle modifying general arousal effects in the rat olfactory bulb. Electroencephalography \& Clinical Neurophysiology, 1979, 46, 665-674.

Gilbert, C. D., \& Kelly, J. P. The projections of cells in different layers of the cat's visual cortex. Journal of Comparative Neurology, 1975, 163, 81-105.

Glenn, J. F., \& OAtman, L. C. Effects of visual attention on the latency of auditory evoked potentials. Experimental Neurology, $1977,57,34-40$.

Goldberg, M., \& Robinson, D. L. Visual system: Superior colliculus. In R. B. Masterton (Ed.), Handbook of behavioral neurobiology (Vol. 1): Sensory integration. New York: Plenum Press, 1978.

Goldberg, S., \& Galin, M. A. Responses of retinal ganglion cell axons to lesions in the adult mouse retina. Investigative Ophthalmology, 1973, 12, 382-385.

Granit, R. Receptors and sensory perception. New Haven, Conn: Yale University Press, 1955.

Granit, R. Neural activity in the retina. In J. Field (Ed.), Handbook of physiology (Sec. 1, Vol. 1): Neurophysiology. Washington D.C: American Physiological Society, 1959.

Halpern, M., Wang, R. T., \& Colman, D. R. Centrifugal fibers to the eye in a nonavian vertebrate: Source revealed by horseradish peroxidase studies. Science, 1976, 194, 1185-1188.

Harter, M. R., \& Aine, C., \& Schroeder, C. Hemispheric differences in the neural processing of stimulus location and type: Effects of selective attention on visual evoked potentials. Neuropsychologia, 1982, 20, 421-437.

Harter, M. R., \& Salmon, L. E. Intra-modality selective attention and evoked cortical potentials to randomly presented patterns. Electroencephalography \& Clinical Neurophysiology, 1972, 83, 605-613.

Hernandez-Peon, R., Scherrer, H., \& Jouvet, M. Modification of electrical activity in cochlear nucleus during "attention" in unanesthetized cats. Science, 1956, 123, 331-332.

Hillyard, S. A., Hink, R. F., Schwent, V. L., \& Picton, T. W. Electrical signs of selective attention in the human brain. Science, 1973, 182, 177-180.

Honrubia, F. M., \& ElliotT, J. H. Efferent innervation of the retina. I. Morphological study of the human brain. Archives of Ophthalmology, 1968, 80, 98-103.

Jacobson, J. H., \& Gestring, G. F. Centrifugal influence on the electroretinogram. Annals of the New York Academy of Science, 1958, 74, 362-371.

KERR, D. I. B., \& HAGBARTH, K. E. An investigation of olfactory centrifugal fiber system. Journal of Neurophysiology, 1955, 18, 362-374.

KLINKE, R., \& G ALLEY, N. Efferent innervation of vestibular and auditory receptors. Physiological Reviews, 1974, 54, 316-357.

Kuffler, S. W., Nicholls, J. G., \& Orkand, R. K. Physiological properties of glial cells in the central nervous system of amphibia. Journal of Neurophysiology, 1966, 29, 768-787.

Lamarre, Y., Filion, M., \& Cordeau, J. P. Neuronal discharges of the ventrolateral nucleus of the thalamus during sleep and wakefulness in the cat. I. Spontaneous activity. Experimental Research, 1971, 12, 480-496.

Lindsley, D. B. Attention, consciousness, sleep and wakefulness. In J. Field (Ed.), Handbook of physiology (Sec. 1, Vol. 1): Neurophysiology. Washington, D.C: American Physiological Society, 1959.

LinDSLEY, D. B. The reticular activating system and perceptual integration. In D. Sheer (Ed.), Electrical stimulation of the brain. Austin: University of Texas Press, 1961.

Livingston, R. B. Central control of receptors and sensory transmission systems. In J. Field (Ed.), Handbook of physiology (Sec. 1, Vol. 1): Neurophysiology. Washington, D.C: American Physiological Society, 1959.

Livingston, R. B. Sensory processing, perception and behavior. New York: Raven Press, 1978.

LuKas, J. H. Human auditory attention: The olivocochlear bundle may function as a peripheral filter. Psychophysiology, $1980,17,444-452$.

LuKas, J. H. The role of efferent inhibition in human auditory attention: An examination of the auditory brainstem potentials. International Journal of Neuroscience, 1981, 12, 137-145.

Marchiafava, P. L. Centrifugal actions on amacrine and ganglion cells in the retina of the turtle. Journal of Physiology (London), 1976, 255, 137-155.

MatThews, P. B. C. Muscle spindles and their motor control Physiology Review, 1964, 44, 219-288.

McCallum, W. C., Curry, S. H., Cooper, R., Pocock, P. V., \& Papakostopoulos, D. Brain event-related potentials as indicators of early selective processes in auditory target localization. Psychophysiology, 1983, 20, 1-17.

MiLes, F. A. Centrifugal control of avian retina. III. Effects of electrical stimulation of the isthmo-optic tract on receptive field properties of retinal ganglion cells. Brain Research, 1972, 48 , 115-129. (a)

MiLes, F. A. Centrifugal control of avian retina. IV. Effects of reversible cold block of the isthmo-optic tract on receptive field properties of cells in the retina and isthmo-optic nucleus. Brain Research, 1972, 48, 139-145. (b)

Miller, R. F., Dacheux, R., \& Proenza, L. Müller cell depolarization evoked by antidromic optic nerve stimulation. Brain Research, 1977, 121, 162-166.

Miller, R. F., \& Dowling, J. E. Intracellular responses of the Müller (glial) cells of mudpuppy retina: Their relation to b-wave of the electroretinogram. Journal of Neurophysiology, 1970, 33, 323-341.

Mirsky, A. F. Attention-A commentary. In E. Callaway, P. Tueting, \& S. H. Koslow (Eds.), Event-related brain potentials in man. New York: Academic Press, 1978.

Mirsky, A. F., Bloch, S., Tecce, J. J., Lessell, S., \& Marcus, E. Visual evoked potentials during experimentally induced spike-wave activity in monkeys. Electroencephalography \& Clinical Neurophysiology, 1973, 35, 25-37.

Moruzzi, G., \& Magoun, H. W. Brain stem reticular formation and activation of the EEG. Electroencephalography \& Clinical Neurophysiology, 1949, 1, 455-473.

NÄÄtÄnEN, R. Selective attention and evoked potentials in humans-A critical review. Biological Psychology, 1975, 2, 237-307. 
NäÄtÄnen, R. Processing negativity: An evoked-potential reflection of selective attention. Psychological Bulletin, 1982, 92, 605-640.

NaUta, W. J. H. Hippocampal projections and related neural pathways to the midbrain in the cat. Brain, 1958, 81, 319-340.

Negishi, K. E., Lu, S., \& Verzeano, M. Neuronal activity in the lateral geniculate body and the nucleus reticularis of the thalamus. Vision Research, 1962, 1, 343-353.

Noback, C. R., \& Mettler, F. Centrifugal fibers to the retina in the rhesus monkey. Brain, Behavior, and Evolution, 1973, 7, 382-399.

OAtman, L. C. Role of visual attention on auditory evoked potentials in unanesthetized cats. Experimental Neurology, 1971, 32, 341-356.

OAtman, L. C. Effects of visual attention on the intensity of auditory evoked potentials. Experimental Neurology, 1976, 51, 41-53.

OAtman, L. C. Spectral analysis of cortical EEG activity during visual attention. Physiological Psychology, 1982, 10, 336-342.

OAtman, L. C., \& Anderson, B. W. Effects of visual attention on tone burst evoked auditory potentials. Experimental Neurology, 1977, 57, 200-211.

OAtman, L. C., \& Anderson, B. W. Suppression of the auditory frequency following response during visual attention. Electroencephalography \& Clinical Neurophysiology, 1980, 49, 314-322.

Ogden, T. E. On the function of efferent retinal fibres. In C. Von Euler, S. Skowland, \& V. Soderberg (Eds.), Structure and function of inhibitory neuronal mechanisms. New York: Pergamon Press, 1968.

Oleson, T. D., Ashe, J. H., \& Weinberger, N. M. Modification of auditory and somatosensory system activity during pupillary and conditioning in the paralyzed cat. Journal of Neurophysiology, 1975, 38, 1114-1139.

Picton, T. W., \& Hillyard, S. A. Human auditory evoked potentials. II. Effects of attention. Electroencephalography \& Chinical Neurophysiology, 1974, 36, 191-199.

Picton, T. W., Hillyard, S. A., Galambos, R., \& Schiff, M. Human auditory attention: A central of peripheral process? Science, 1971, 173, 351-353.

Picton, T. W., Hillyard, S. A., Krausz, H. I., \& Galambos, R. Human auditory evoked potentials. I: Evaluation of components. Electroencephalography \& Clinical Neurophysiology, 1974, 36, 179-190.

Picton, T. W., Stapells, D. R., \& Campbell, K. B. Auditory evoked potentials from the human cochlea and brainstem. Journal of Otolaryngology, 1981, 10(Suppl. 9), 1-41.

Polyak, S. The vertebrate visual system. Chicago: University of Chicago Press, 1957.

Posner, M. I., Nissen, M. J., \& Ogden, W. C. Attended and unattended processing modes: The role of set for spatial location. In H. L. Pick (Ed.), Modes of perception. Hillsdale, N.J: Erlbaum, 1977.

Rasmussen, G. L. Efferent fibers of the cochlear nerve and cochlear nucleus. In G. L. Rasmussen \& W. F. Windle (Eds.), Neural mechanisms of the auditory and vestibular systems. Springfield, Ill: Thomas, 1960.

Rasmussen, G. L. Anatomic relationships of the ascending and descending auditory systems. In W. S. Fields \& B. R. Alford (Eds.), Neurological aspects of auditory and vestibular disorders. Springfield, Ill: Thomas, 1964.

Rodieck, R. W. The vertebrate retina. San Francisco: Freeman, 1973.

Rodieck, R. W. Visual pathways. Annual Review of Neuroscience, 1979, 2, 193-225.

Sacks, J. G., \& Lindenbera, R. Efferent nerve fibers in the anterior visual pathways in bilateral congenital cystic eyeballs. American Journal of Ophthalmology, 1969, 68, 691-695.

SCHE IBEL, M. E., \& SCHEIBEL, A. B. The organization of nucleus reticularis thalami: A Golgi study. Brain Research, 1966, 1, 43-62.

Scheibel, M. E., \& Scheibel, A. B. Structural organization of nonspecific thalamic nuclei and their projection toward cortex. Brain Research, 1967, 6, 60-94.

Schmidt, R. Control of the access of afferent activity to somatosensory pathways. In A. Iggo (Ed.), Handbook of sensory physiology (Vol. 2): Somatosensory system. Berlin: Springer, 1973.

Shepherd, G. M. The olfactory bulb as a simple cortical system: Experimental analysis and functional implications. In F. $O$. Schmitt (Ed.), The neurosciences second study program. New York: Rockefeller University Press, 1970.

Shortess, G. K. Some comments on the functional significance of centrifugal fibers to the vertebrate retina. In J. C. Armington, J. Krauskopf, \& B. R. Wooten (Eds.), Visual psychophysics and physiology. New York: Academic Press, 1978.

Singer, W. Control of thalamic transmission by corticofugal and ascending reticular pathways in the visual system. Physiological Reviews, 1977, 57, 386-420.

Skinner, J. E., \& Yingling, C. D. Central gating mechanisms that regulate event-related potentials and behavior. In J. E. Desmedt (Ed.), Attention, voluntary contraction and eventrelated cerebral potentials: Progress in clinical neurophysiology (Vol. 1). Basel: Karger, 1977.

Spinelli, O. N., Pribram, K. H., \& Weingarten, M. Centrifugal optic nerve responses evoked by auditory and somatic stimulation. Experimental Neurology, 1965, 12, 303-319.

Starr, A., \& Wernick, J. S. Olivocochlear bundle stimulation: Effects on spontaneous and tone-evoked activities of single units in cat cochlear nucleus. Journal of Neurophysiology, 1968, 31, 549-564.

Van Hassalt, P. The centrifugal control of retinal function. A review. Ophthalmological Research, 1972/73, 4, 298-320.

Van Voorhis, S., \& Hillyard, S. Visual evoked potentials and selective attention to points in space. Perception \& Psychophysics, 1977, 22, 54-62.

Wiederhold, M. L., \& Kiang, N. Y. S. Effects of electrical stimulation of the crossed olivo-cochlear bundle on single auditory nerve fibers in the cat. Journal of the Acoustical Society of America, 1970, 48, 950-965.

Wilson, M. Visual system: Pulvinar-extrastriate cortex. In R. B. Masterton (Ed.), Handbook of behavioral neurobiology (Vol. 1): Sensory integration. New York: Plenum Press, 1978.

Wolter, J. R. The centrifugal nerves in the human optic tract, chiasm, optic nerve, and retina. Transactions of the American Ophthalmological Society, 1965, 63, 678-707.

WOLTER, J. R., \& LUND, O. E. Reaction of centrifugal nerves in the human retina. American Journal of Ophthalmology, 1968, 66, 222-232.

Wurtz, R. H., \& Albano, J. E. Visual-motor function of the primate superior colliculus. Annual Review of Neuroscience, $1980,3,189-226$.

Yingling, C. D., \& Skinner, J. E. Selective regulation of thalamic sensory relay nuclei by nucleus reticularis thalami. Electroencephalography \& Clinical Neurophysiology, 1976, 41, 476-482.

\section{NOTES}

1. The term "component" is used in this paper to denote specific deflections contained in raw averaged VEPs. It is not to be confused with the use of the term for designating deflections abstracted by factor analysis from averaged VEPs.

2. Observations made in relation to an earlier ERG study performed by the authors (Eason, Flowers, \& Oakley, 1983) revealed that the choice of earlobe as a reference site (right or left) had no discernible effect on the magnitude or waveform of retinal responses measured at the active electrode sites, including canthal sites. Thus, the right earlobe was used throughout the study.

(Manuscript received February 3, 1983; accepted for publication February 24, 1983.) 